\title{
On Limiting the Lateral Expansion of the Cloud of Fragments of a Destructed Meteoroid
}

\author{
I.G. Brykina ${ }^{1}$, M.D. Bragin ${ }^{2,3}$ \\ ${ }^{1}$ Institute of Mechanics of Lomonosov Moscow State University, \\ Moscow, 119192, Russia \\ ${ }^{2}$ Keldysh Institute of Applied Mathematics, \\ Moscow, 125047, Russia \\ ${ }^{3}$ Moscow Institute of Physics and Technology, \\ Dolgoprudny, Moscow Region, 141701, Russia \\ shantii@mail.ru
}

\begin{abstract}
The motion and ablation of a meteoroid breaking up into a large number of fragments are considered. At the first stage, fragments move with a common shock wave, before dispersing enough distance to move independently. We consider models of cloud of fragments that simulate the meteoroid disruption at this stage: the two-parameter model, which takes into account changes in the shape and density of the cloud, and simple models used in the literature that do not take into account these effects. Models differ in equations for the rate of cloud lateral expansion. The unrealistically strong increase in the midsection radius, which is given by simple models, is usually limited in the literature to a certain specified value. The effect of this midsection radius cutoff in different fragment cloud models on the results of modeling the energy deposition of the Chelyabinsk superbolide is studied. For this purpose, the equations of the physical theory of meteors are solved numerically using the same ablation model developed by the authors for different fragmentation models. The influence of the heat transfer coefficient on the energy deposition of the bolide obtained using different fragment cloud models and on the applicability of these models is studied.
\end{abstract}

Keywords: meteoroid, cloud of fragments, heat transfer coefficient, energy deposition.

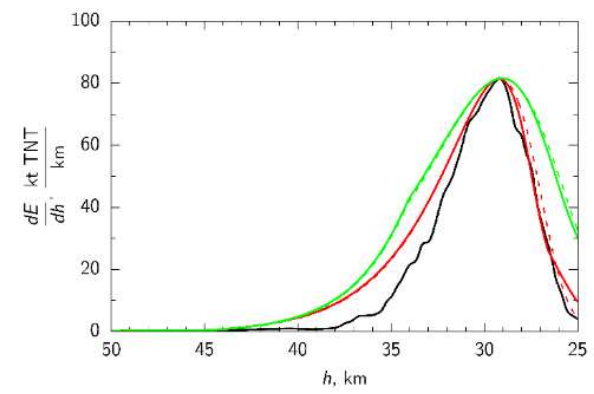

$a$

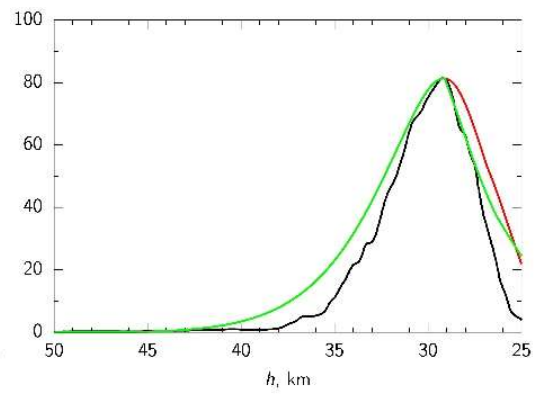

b

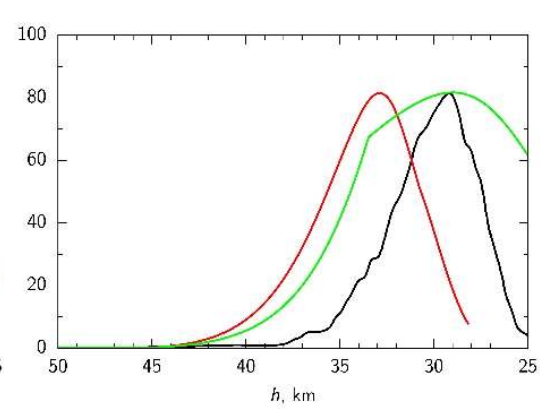

c

Energy deposition of the Chelyabinsk bolide without midsection radius cutoff (red curves) and with best cutoff (green) for different fragmentation models. Black curve - observational data [17].

a) Two-parameter model. Solid and dashed curves correspond to formula (7) for the heat transfer coefficient $C_{H}$ with uncertainty parameter $\psi=1$ and to $C_{H}=0.1$;

b) Simple model $[2,6]$ with coefficient $c=1$ in equation (2); parameter $\psi=0.3$;

c) Simple model [5] with coefficient $c=(7 / 2)^{1 / 2}$ in equation (2); parameter $\psi=0.3$ 


\title{
Об ограничении бокового расширения облака фрагментов разрушенного метеороида
}

\author{
И.Г. Брыкина ${ }^{1}$, М.Д. Брагин ${ }^{2,3}$ \\ ${ }^{1}$ НИИ механики МГУ им. М.В. Ломоносова, \\ Россия, Москва, 119192, Мичуринский пр., 1 \\ ${ }^{2}$ ИПМ им. М.В. Келдыша РАН, \\ Россия, Москва, 125047, Миусская пл., 4 \\ ${ }^{3}$ Московский физико-технический институт, \\ Россия, Долгопрудный, 141701, Институтский n., 7 \\ shantii@mail.ru
}

\begin{abstract}
Аннотация
Рассматривается движение и абляция метеороида, дробящегося на большое количество осколков. На первом этапе фрагменты движутся с общей ударной волной, прежде чем разойтись на расстояние, достаточное для независимого движения. Рассматриваются модели облака фрагментов, моделирующие разрушение метеороида на данном этапе: двухпараметрическая модель, учитывающая изменения формы и плотности облака, и простые модели, используемые в литературе, не учитывающие эти эффекты. Модели отличаются уравнениями для скорости бокового расширения облака. Нереально сильное возрастание радиуса миделя, которое дают простые модели, в литературе принято ограничивать некоторым заданным значением. Исследуется влияние такого обрезания радиуса миделя в разных моделях облака фрагментов на результаты моделирования энерговыделения Челябинского суперболида. Для этого уравнения физической теории метеоров решаются численно с использованием одной и той же, разработанной авторами, модели абляции для разных моделей фрагментации. Изучается влияние коэффициента теплопередачи на энерговыделение болида, полученное с помощью разных моделей облака фрагментов, и на применимость этих моделей.
\end{abstract}

Ключевые слова: метеороид, облако фрагментов, коэффициент теплопередачи, энерговыделение.

\section{1. Введение}

Разрушение - это один из основных процессов, наряду с абляцией, оказывающих существенное влияние на взаимодействие космического тела с атмосферой. Разрушение космических тел, входящих в атмосферу Земли с различными скоростями, составами, структурами, размерами и прочностью, может происходить по-разному, поэтому существуют разные подходы к моделированию их фрагментации. В том случае, когда метеороид распадается на большое количество фрагментов, на первом этапе они движутся с общей ударной волной как единое тело. На втором этапе фрагменты разлетаются на достаточное расстояние, чтобы иметь свои собственные ударные волны, которые взаимодействуют друг с другом. На третьем этапе, после полного разделения фрагментов, они движутся самостоятельно. Разные стадии движения фрагментов изучались Максимовым [1]. Обычно при построении моделей разрушения метеороидов вторая стадия движения осколков с взаимодействием ударных волн игнорируется, и в большинстве исследований рассматривается независимое движение фрагментов. Для моделирования разрушения метеороида на первом этапе предлагались, использовались и используются до настоящего времени модели облака фрагментов, движущихся 
как единый объект [2-11 и др.]. Такое облако фрагментов под действием сил давления деформируется (сплющивается): оно сжимается в направлении полета и расширяется в боковом направлении. Модели по существу отличаются уравнением, описывающим боковое расширение облака фрагментов, т.е. уравнением для скорости возрастания радиуса миделя раздробленного метеороида. Модели облака фрагментов могут быть применены к метеороидам размером более нескольких метров, которые интенсивно разрушаются.

В данной работе, продолжающей исследования [12], рассматриваются модели облака фрагментов, моделирующие разрушение метеороида на первом этапе: двухпараметрическая модель, развитая авторами [9], учитывающая изменения формы и плотности облака, и простые модели, не учитывающие эти эффекты: самая первая из такого рода моделей, предложенная С.С. Григоряном [2], и общепринятая, наиболее часто используемая в литературе [7, 8, 10, 11 и др.], модель Хиллза и Годы [5]. Простые модели дают физически нереальное сильное возрастание радиуса миделя облака фрагментов, поэтому в литературе принято ограничивать его некоторым значением, обычно не превышающим $7 \div 8[8,10,11]$. Исследуется влияние такого обрезания радиуса миделя на результаты моделирования энерговыделения Челябинского суперболида с помощью разных моделей фрагментации. Для этого проводятся численные расчеты уравнений физической теории метеоров с использованием одной и той же модели абляции, развитой авторами, и одного и того же коэффициента сопротивления, также полученного авторами, для разных моделей фрагментации. Изучается влияние коэффициента теплопередачи на результаты расчетов энерговыделения болида и на применимость моделей облака фрагментов, которая определяется путем сопоставления с наблюдательной кривой энерговыделения.

\section{2. Модели облака фрагментов}

Предполагается, что до начала разрушения метеороид движется как единое тело (шар), затем он продолжает свой полет как облако фрагментов и паров, объединенных общей ударной волной, при этом шар трансформируется в сфероид с отношением полуосей $b / a=k \geq 1$. В двухпараметрической модели [9], кроме параметра сплющивания $k$, вводится параметр $\gamma$ $(\gamma \leq 3)$, характеризующий уменьшение плотности раздробленного метеороида $\delta$ из-за увеличения промежутков между фрагментами: $\delta=\delta_{e} / \gamma^{3}, \delta_{e}$ - его начальная плотность.

В двухпараметрической модели уравнение для скорости увеличения радиуса миделя облака фрагментов $R_{S}$ имеет вид

$$
\begin{gathered}
\frac{\mathrm{d} R_{S}}{\mathrm{~d} t}=\left(\frac{\gamma^{3}}{k}\right)^{1 / 2}\left(\frac{\rho}{\delta_{e}}\right)^{1 / 2} V, \quad k=\frac{4 \pi \delta_{e}}{3} \frac{R_{S}^{3}}{M \gamma^{3}}, \\
\gamma=1+\frac{\rho^{1 / 2}-\rho_{f}^{1 / 2}}{\rho_{m}^{1 / 2}-\rho_{f}^{1 / 2}}\left(\gamma_{m}-1\right)
\end{gathered}
$$

Здесь $t$ - время; $\rho$ - плотность атмосферы; $V$ и $M$ - скорость и масса метеороида; индексы $f$ и $m$ соответствуют значениям величин на высоте начала фрагментации $h_{f}$ и высоте максимальной яркости болида $h_{m}$. Параметр $\gamma_{m}$ определяется приравниваем расчетной и наблюдаемой высот $h_{m}$. Подробный вывод уравнений $(1)$ и описание модели даны в $[9,12]$.

В простых моделях уравнения для радиуса миделя $R_{S}$ получены без учета изменения плотности и формы метеороида (фактически для шара) и имеют вид

$$
\frac{\mathrm{d} R_{S}}{\mathrm{~d} t}=c\left(\frac{\rho}{\delta_{e}}\right)^{1 / 2} V,
$$


где $c=$ const : $c=1$ в модели Григоряна $[2,6]$ и $c=(7 / 2)^{1 / 2} \approx 1.87$ в модели Хиллза и Годы [5]. Постоянность коэффициента $c$ в уравнении (2) дает возможность найти его аналитическое решение в случае прямолинейной траектории и изотермической атмосферы. Используя соотношение

$$
\frac{\mathrm{d}}{\mathrm{d} t}=\frac{\rho V \sin \theta}{h^{*}} \frac{\mathrm{d}}{\mathrm{d} \rho}
$$

где $\theta$ - угол входа метеороида в атмосферу по отношению к горизонту; $h^{*}$ - шкала высот, принимаемая равной 7 км, нетрудно получить

$$
R_{S}=R_{f}\left[1+\frac{2 c h^{*}}{\sin \theta \delta_{e}^{1 / 2} R_{f}}\left(\rho^{1 / 2}-\rho_{f}^{1 / 2}\right)\right]
$$

Здесь $R_{f}$ - радиус тела на высоте $h_{f}$. Из выражения (3) следует, что значение радиуса $R_{S}$ в простых моделях определяется не текущими, а только начальными параметрами на высоте фрагментации: $\theta, \rho_{f}, R_{f}$ и $\delta_{e}$, нет влияния абляции, т.е. изменения массы метеороида, на $R_{S}$.

Это означает, что при применении простых моделей задача фрагментации метеороида отделена от задачи его абляции и движения. В этом заключено несоответствие: абляция влияет на массу метеороида и не влияет на его радиус миделя. В двухпараметрической модели скорость роста $R_{S}$ зависит от массы $M$, которая меняется из-за абляции, поэтому, чтобы найти $R_{S}$ и $M$, надо решать совместную задачу фрагментации, абляции и торможения метеороида. В этой модели учитывается также уменьшение плотности облака фрагментов (влияние $\gamma$ ) и изменение его формы (влияние $k$ ), а в простых моделях - нет. В этом тоже есть некоторое несоответствие: в работе [5] и в последующих работах, использующих модель [5], говорится об облаке осколков и промежутках между ними, однако плотность облака осколков принимается постоянной и равной плотности тела до начала дробления.

\section{3. Постановка задачи. Уравнения и начальные условия}

Для расчета изменения скорости, массы и кинетической энергии метеороида вдоль траектории используются уравнения торможения и абляции, прямолинейной траектории и изотермической атмосферы [12]

$$
\begin{gathered}
M \frac{\mathrm{d} V}{\mathrm{~d} t}=-\frac{\pi}{2} R_{s}^{2} C_{D} \rho V^{2}, \quad Q \frac{\mathrm{d} M}{\mathrm{~d} t}=-\frac{\pi}{2} R_{s}^{2} C_{H} \rho V^{3}, \\
\frac{\mathrm{d} h}{\mathrm{~d} t}=-V \sin \theta, \quad \rho=\rho_{0} \exp \left(-\frac{h}{h^{*}}\right)
\end{gathered}
$$

Здесь $C_{D}$ и $C_{H}$ - коэффициенты сопротивления и теплопередачи на единицу площади миделева сечения; $Q$ - эффективная теплота уноса массы; $h$ - высота; $\rho_{0}=1.225 \times 10^{3}$ г/ $\mathrm{cm}^{3}$.

Изменение кинетической энергии метеороида $E$ в единицу времени $\mathrm{d} E / \mathrm{d} t$ и энерговыделение на единицу высоты $\mathrm{d} E / \mathrm{d} h$ определяются по формулам

$$
\frac{\mathrm{d} E}{\mathrm{~d} h}=\frac{J}{V \sin \theta}, \quad J=-\frac{\mathrm{d} E}{\mathrm{~d} t}=-\left(\frac{V^{2}}{2} \frac{\mathrm{d} M}{\mathrm{~d} t}+M V \frac{\mathrm{d} V}{\mathrm{~d} t}\right)
$$

При применении простых моделей фрагментации уравнения (4) решаются вместе с уравнением (2) или (3), а для двухпараметрической модели решаются уравнения (1) и (4), до начала дробления полагается $\gamma=1$ и $k=1$. Для решения полученной системы уравнений необходимо знать коэффициент лобового сопротивления $C_{D}$ и коэффициент теплопередачи $C_{H}$. Коэффициент сопротивления сфероида полагался равным 


$$
C_{D}=1.78-\frac{0.85}{k}
$$

Эта формула получена путем аппроксимации численных расчетов [13] гиперзвукового потока диссоциированного воздуха около космических аппаратов со сфероидальной лобовой поверхностью и согласуется с точностью до $12 \%$ с аналитическим решением [9], полученным для сфероида при задании распределения давления по формуле Ньютона.

Для коэффициента радиационной теплопередачи $C_{H}$ для сфероида использовалась аппроксимационная формула

$$
C_{H}=\psi \varphi(V, k) \beta(k) C_{H_{0}}(V, R, \rho),
$$

где $C_{H_{0}}$ - коэффициент теплопередачи в точке торможения - определялся по формуле работы [14] в зависимости от значений $V, R, \rho$ ( $R$ - радиус затупления тела); параметр $\beta(k)$ характеризует зависимость теплового потока в точке торможения сфероида от параметра $k$ [15]; параметр $\varphi(V, k)$ - изменение его вдоль поверхности; $\beta$ и $\varphi$ приведены в [9].

В выражение для коэффициента теплопередачи (7) введен фактор неопределенности $\psi$ для того, чтобы учесть влияние на радиационный поток к телу опережающего излучения, экранирования его парами метеороида, турбулентности, неопределенность в оптических свойствах горячего воздуха и паров, в моделях переноса излучения и поля течения, и другие неизвестные факторы. При исследовании неопределенности радиационного нагрева ударного слоя было показано [16], что неопределенность радиационного теплового потока в точке торможения составляет примерно от +81 до $-52 \%$ для входа в атмосферу Земли со скоростью $15 \mathrm{kм} / \mathrm{c}$. Для изучения влияния неопределенности коэффициента теплопередачи $C_{H}$ на характеристики взаимодействия метеороида с атмосферой и на применимость разных моделей облака фрагментов параметр $\psi$ варьировался при проведении расчетов. Расчеты также проводились с постоянным значением $C_{H}$, принятым в литературе.

Для расчета коэффициентов сопротивления и теплопередачи по формулам (6) и (7) при использовании простых моделей фрагментации нужно знать параметр $k$, который непосредственно не входит в эти модели. Параметр формы $k$ вычислялся из второго соотношения (1), связывающего массу и радиус миделя сфероида. Заметим, что в модели [5] Хиллза и Годы облако фрагментов также рассматривается как сфероид.

Двухпараметрическая модель, простые модели [2, 6] и [5], а также соответствующие им модели с обрезанием радиуса миделя применялись для моделирования энерговыделения Челябинского болида с целью сопоставить возможности моделей воспроизвести наблюдательную кривую энерговыделения [17]. Обыкновенные дифференциальные уравнения для каждой из моделей решались методом Рунге-Кутты.

В качестве начальных параметров, соответствующих входу Челябинского метеороида в атмосферу Земли, брались результаты обработки наблюдательных данных [18]: $V_{e}=19$ км/с, $\theta=18^{\circ}, \delta_{e}=3.3$ г $/ \mathrm{cm}^{3}$, высота начала фрагментации $h_{f}$ полагалась равной 45 км, что соответствует начальной прочности метеороида $\sigma=0.75 \mathrm{MПа;} \mathrm{эффективная} \mathrm{теп-}$ лота уноса массы $Q$ полагалась равной $6 \mathrm{~km}^{2} / \mathrm{c}^{2}$. Неизвестная начальная масса метеороида $M_{e}$ для каждой из моделей и каждого расчетного случая определялась таким образом, чтобы максимальное значение энерговыделения совпало с наблюдательным значением [17].

Отметим, что разрушение Челябинского метеороида - очень сложное явление, и, конечно, простые модели облака фрагментов не дают полного представления о его дроблении. Отдельные фрагменты отделялись от облака и вели себя независимо, мелкие фрагменты тормозились и падали на землю. Однако, принимая во внимание, что самые крупные наблюдаемые фрагменты отделились от облака на высоте 25 км и ниже [18], есть основание использовать модели облака фрагментов при моделировании взаимодействия Челябинского метеороида с атмосферой выше этой высоты. 


\section{4. Результаты расчетов}

Расчеты для различных моделей фрагментации проводились при разных значениях коэффициента теплопередачи $C_{H}$, а именно, варьировался параметр $\psi$ в формуле (7) и проводились расчеты при разных постоянных значениях $C_{H}$. Фактически, $C_{H}$ - это единственный свободный параметр, который можно менять (в разумных пределах) в силу его неопределенности.

Для двухпараметрической модели варьирование параметра $\psi$ ведет только к изменению формы кривой энерговыделения, делая ее шире при уменьшении значения коэффициента теплопередачи, и к некоторому изменению оценки начальной массы, но не влияет на расчетную высоту максимальной яркости болида. Это связано с тем, что корректирование имеющегося в этой модели параметра $\gamma_{m}$ позволяет достичь совпадения расчетной высоты максимума энерговыделения $h_{m}$ с наблюдательной. Расчеты показали, что для двухпараметрической модели наилучшее согласование с наблюдательной кривой энерговыделения Челябинского суперболида [17] получается при $\psi=1$ и $C_{H}=0.1$; при этом модель дает значение начальной массы $M_{e}=1.325 \times 10^{10}$ г, близкое к наиболее вероятным значениям 1.2 и $1.3 \times 10^{10}$ г по оценкам работ [18] и [19]. Наблюдательная и расчетные для двухпараметрической модели кривые энерговыделения вдоль траектории приведены на рис. 1 в зависимости от высоты полета $h$. На этом же рисунке приведены кривые изменения радиуса миделя раздробленного метеороида, отнесенного к его значению $R_{e}$ при входе в атмосферу, характеризующие боковое расширение облака фрагментов.
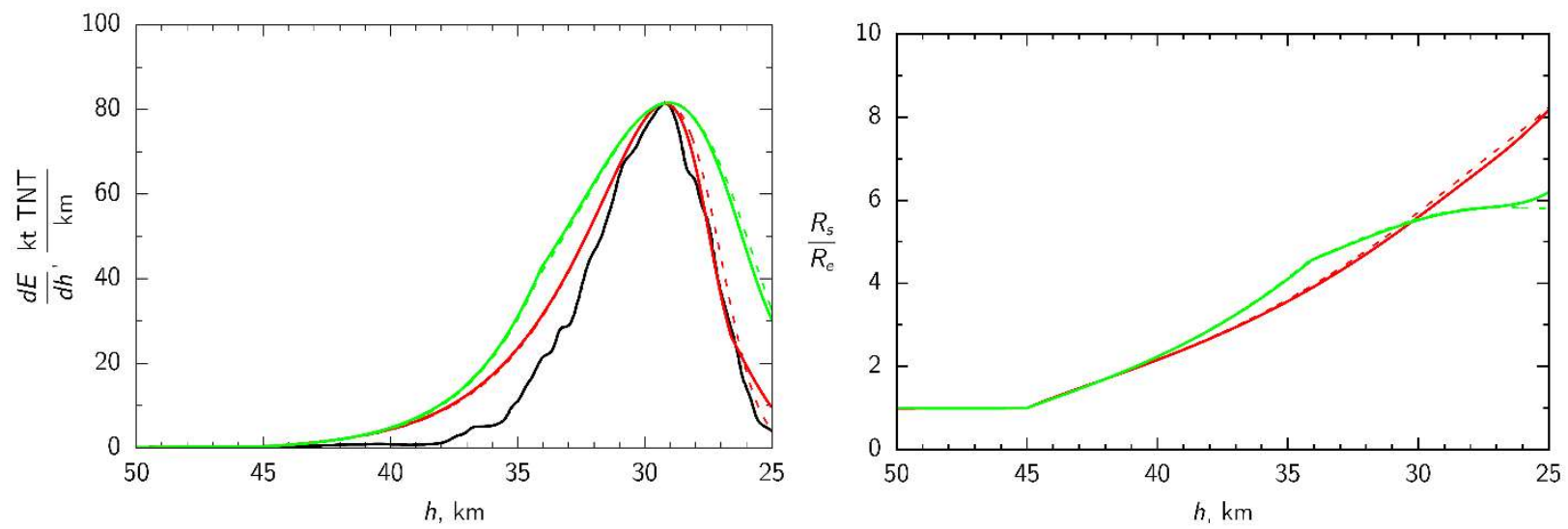

Рис. 1. Энерговыделение метеороида и изменение его радиуса миделя для двухпараметрической модели без обрезания (красные кривые) и с обрезанием (зеленые). Сплошные и штриховые кривые соответствуют $\psi=1$ в формуле (7) и $C_{H}=0.1$. Черная кривая - наблюдательные данные [17]

На рис. 1 показаны также результаты расчетов для двухпараметрической модели с обрезанием: рост параметра сплющивания $k$ ограничивается обрезанием его при некотором значении $k^{*}$, в данном случае $k^{*}$ полагалось равным 10 . Обрезание роста параметра $k$ для двухпараметрической модели ведет к тому, что радиус миделя несколько уменьшается после прохождения точки траектории, где $k=k^{*}$, однако кривая энерговыделения фрагментированного метеороида становится заметно шире наблюдаемой, причем с уменьшением $k^{*}$ она становится все более широкой и более удаленной от наблюдаемой. Таким образом, для двухпараметрической модели обрезание не имеет смысла, поскольку оно заметно ухудшает согласование с данными наблюдений, а радиус миделя в этой модели увеличивается не так сильно, как в простых моделях.

Для простых моделей варьирование параметра $\psi$ ведет не только к изменению формы кривой энерговыделения метеороида и к изменению его начальной массы, но также и к изменению расчетной высоты максимальной яркости болида. При задании коэффициента теплопередачи $C_{H}$ по формуле (7) при $\psi=1$, а также, при задании постоянного значения 
$C_{H}=0.1$, как принято полагать в большинстве исследований по взаимодействию крупных метеороидов с атмосферой, модель [2,6] дает высоту пика яркости Челябинского болида примерно на 3 километра выше наблюдаемой, а модель [5] - на 6 км выше. Это можно объяснить очень быстрым ростом бокового расширения облака фрагментов в этих моделях и, как следствие, слишком ранним ростом кривой энерговыделения. Уменьшая коэффициент теплопередачи, можно сдвинуть вниз расчетную высоту пика яркости болида. Например, уменьшение $C_{H}$ наполовину $(\psi=0.5)$ сдвигает высоту пика до $\sim 30.5$ км для модели $[2,6]$ (на 1 км выше наблюденной высоты 29.5 км) и до 34 км для модели [5] (на 4.5 км выше). Влияние коэффициента теплопередачи на высоту пика яркости в простых моделях демонстрирует рис. 2.
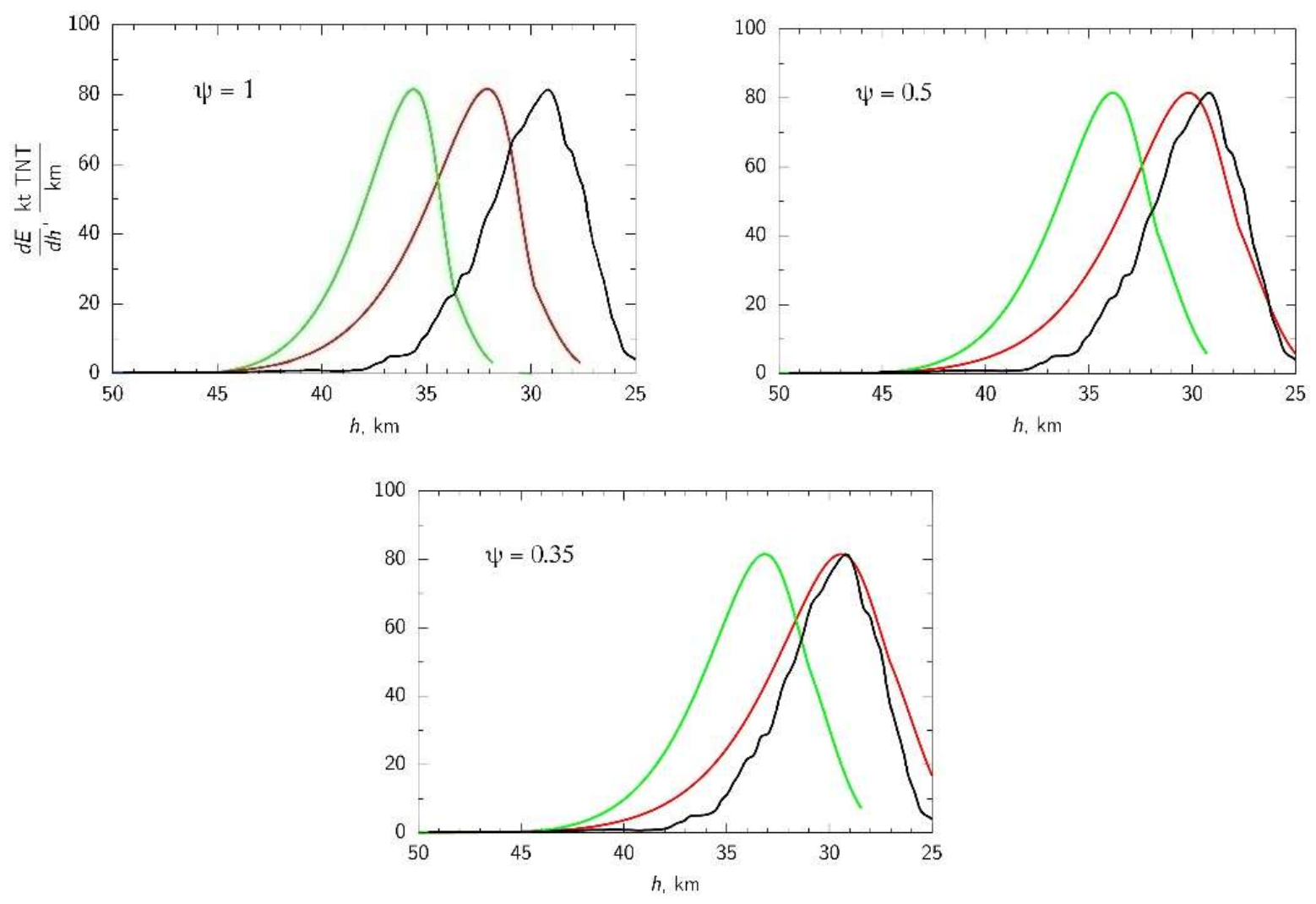

Рис. 2. Влияние коэффициента теплопередачи (параметра $\psi$ ) на энерговыделение метеороида для моделей [2, 6] (красные кривые) и [5] (зеленые); черная кривая - наблюдательная [17]

Для модели Григоряна [2,6] с коэффициентом $c=1$ в уравнении (2) расчетная высота максимума энерговыделения совпадает с наблюденной высотой пика яркости болида при $\psi=0.3$ (и при постоянном значении $C_{H}$, примерно равном 0.03). Кривая энерговыделения для этого случая представлена на рис. 3 , где приведены также наблюдательные данные и кривая изменения радиуса миделя раздробленного метеороида. Модель в этом случае дает значение начальной массы $M_{e}=1.46 \times 10^{10}$ г, что вполне согласуется с оценками работ $[18,19]$. Результаты расчетов с обрезанием радиуса при $R_{S} / R_{e}=8.5$ также показаны на рис. 3 . Это оптимальное значение радиуса обрезания для этой модели, при котором расчетная кривая энерговыделения становится немного ближе к наблюдаемой, чем без обрезания, при этом начальная масса остается примерно такой же. При уменьшении радиуса обрезания кривая энерговыделения становится шире, а ее максимум смещается ниже по высоте, что демонстрирует рис. 4 , где приведены расчеты при радиусе обрезания $R_{S} / R_{e}=7$. Кроме того, растет начальная масса: при радиусе обрезания 7 она увеличивается до $1.986 \times 10^{10} \Gamma$. 

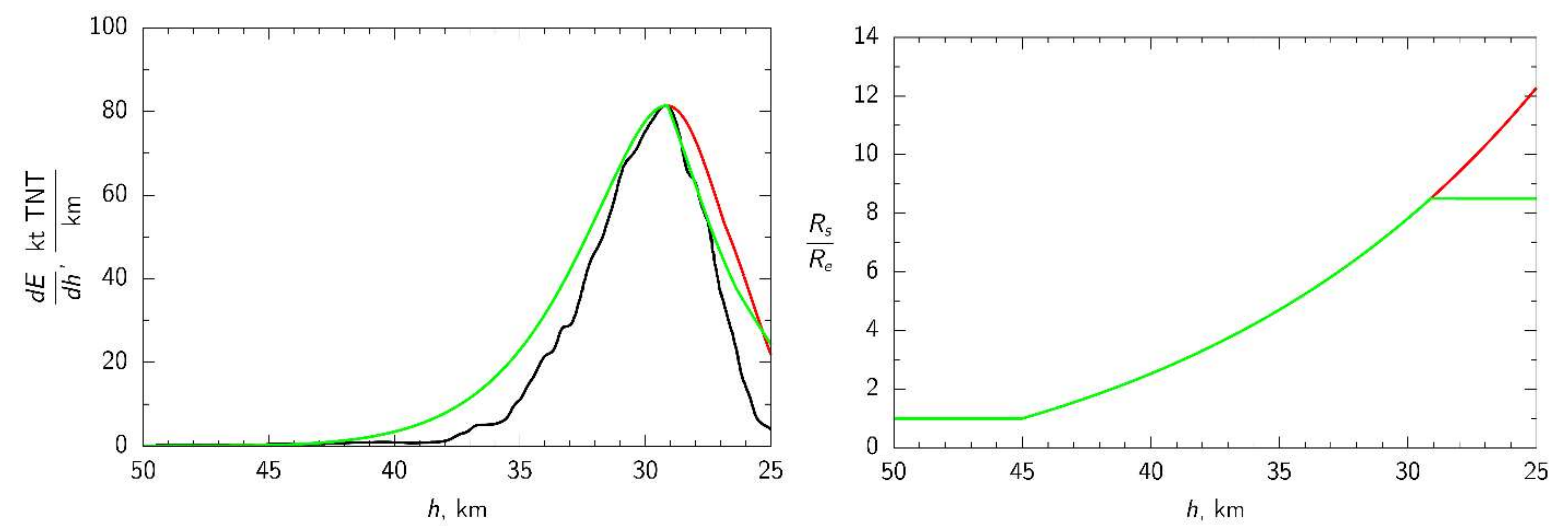

Рис. 3. Энерговыделение метеороида и изменение радиуса миделя для модели $[2,6]$ без обрезания (красные кривые) и с обрезанием при $8.5 R_{e}$ (зеленые). $\psi=0.3$, черная кривая - наблюдательная [17]

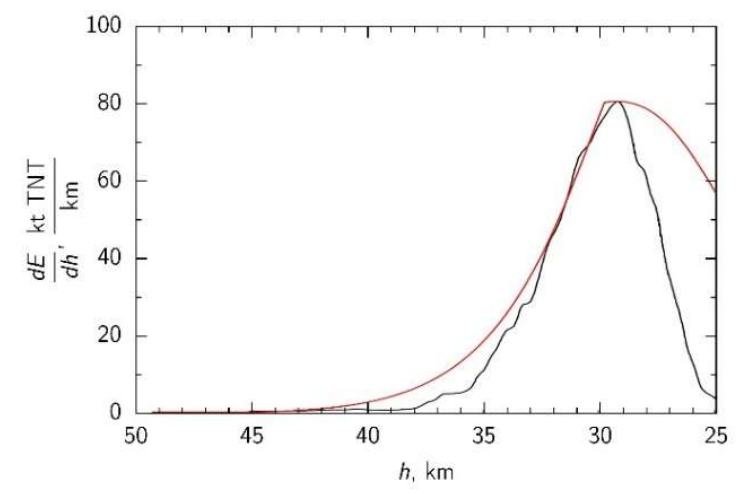

Рис. 4. Энерговыделение метеороида для модели $[2,6]$ с обрезанием при $7 R_{e}$ (красная линия). $\psi=0.3$, черная кривая - наблюдательная [17]

Для модели Хиллза и Годы [5] с коэффициентом $c \approx 1.87$ в уравнении (2) уменьшение коэффициента теплопередачи вплоть до нуля (полное отсутствие абляции) не ведет к совпадению расчетной высоты пика яркости болида с наблюдаемой, при любом значении коэффициента теплопередачи расчетная высота остается выше наблюдаемой. Это объясняется слишком сильным боковым расширением облака фрагментов и быстрым ростом радиуса из-за большого коэффициента $c$ (почти в два раза, превышающего коэффициент $c$ в модели [2, 6]). Обрезание радиуса в модели [5] позволяет сдвинуть высоту пика энерговыделения вниз вплоть до совпадения с наблюдаемой высотой. Наилучший вариант расчета, соответствующий значению радиуса обрезания $7.5 R_{e}$ при $\psi=0.3$, представлен на рис. 5 , где приведены также результаты расчета без обрезания и наблюдательная кривая энерговыделения.
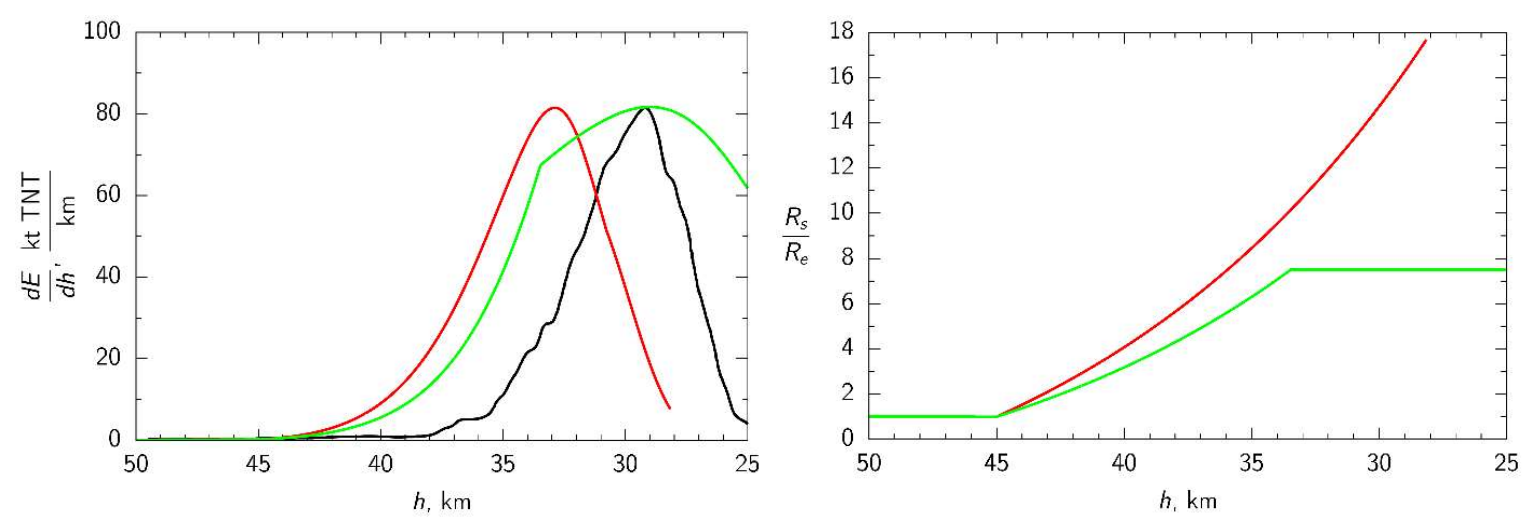

Рис. 5. Энерговыделение метеороида и изменение радиуса миделя для модели [5] без обрезания (красные линии) и с обрезанием при $7.5 R_{e}$ (зеленые). $\psi=0.3$, черная кривая - наблюдательная [17] 
Однако в этом случае совпадения расчетной высоты пика яркости с наблюденной, расчетная кривая энерговыделения оказывается намного шире наблюдательной кривой и значение начальной массы $M_{e}=2.5 \times 10^{10}$ г оказывается намного больше наиболее вероятных значений по оценкам $[18,19]$. При уменьшении радиуса обрезания, как и для модели $[2,6]$, кривая энерговыделения становится шире, а высота ее максимума смещается вниз, что видно из рис. 6 , где показаны результаты расчетов при радиусах обрезания $R_{S} / R_{e}=7$ и 7.5.

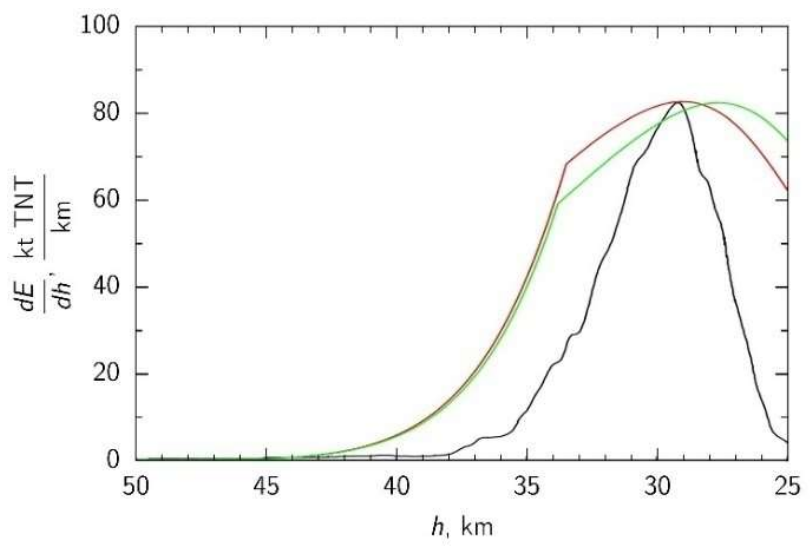

Рис. 6. Энерговыделение метеороида для модели [5] с обрезанием при 7.5R (красная линия) и $7 R_{e}$ (зеленая). $\psi=0.3$, черная кривая - наблюдательная [17]

При этом сам максимум кривой заметно уменьшается, и чтобы компенсировать потерю в максимуме (для соответствия наблюдательному максимуму), нужно увеличивать начальную массу. Так, при радиусе обрезания $7 R_{e}$ модель дает $M_{e}=2.7 \times 10^{10} \Gamma$. Таким образом, при использовании модели фрагментации [5] с коэффициентом $c \approx 1.87$ не удается получить удовлетворительного согласования с наблюдательной кривой энерговыделения как при варьировании коэффициента теплопередачи, так и при варьировании радиуса обрезания.

\section{5. Заключение}

Наилучшее согласование с наблюдательной кривой энерговыделения Челябинского суперболида получается при использовании двухпараметрической модели, учитывающей изменение плотности и формы разрушенного метеороида, при значении параметра $\psi$ в выражении для коэффициента теплопередачи (7), равного 1 и при задании постоянного коэффициента теплопередачи $C_{H}=0.1$. При этом модель дает значение начальной массы метеороида при входе в атмосферу $M_{e}=1.325 \times 10^{10}$ г, близкое к наиболее вероятным значениям 1.2 и $1.3 \times 10^{10}$ г по оценкам $[18,19]$. Ограничение бокового расширения облака фрагментов в случае двухпараметрической модели не имеет смысла, поскольку, во-первых, не улучшает, а ухудшает согласование с наблюдательными данными, а во-вторых, значения радиуса миделя в этой модели принимают вполне допустимые значения, гораздо меньшие, чем в простых моделях, на высотах более 25 км, где модели облака фрагментов применимы.

При использовании простых моделей фрагментации расчетная высота максимальной яркости болида существенно зависит от значения коэффициента теплопередачи. При применении модели $[2,6]$ удовлетворительное согласование с наблюдательной кривой энерговыделения получается при параметре $\psi=0.3$ как без обрезания, так и при обрезании радиуса миделя на значении $8.5 R_{e}$, в обоих случаях модель дает значение начальной массы $M_{e}=1.46 \times 10^{10}$ г. При применении модели фрагментации [5] не удается получить удовлетворительного согласования с наблюдательной кривой энерговыделения при варьировании, как коэффициента теплопередачи, так и радиуса обрезания. 


\section{Благодарности и ссылки на гранты}

Работа выполнена в соответствии с планом исследований НИИ механики МГУ имени М.В. Ломоносова при частичной поддержке гранта РФФИ № 18-01-00740.

\section{Литература}

1. Максимов Ф.А. Сверхзвуковое обтекание системы тел // Компьютерные исследования и моделирование. 2013. № 5. С. 969-980.

2. Григорян С.С. О движении и разрушении метеоритов в атмосферах планет // Космич. исслед. 1979. T. 17. № 6. С. $875-893$.

3. Melosh H.J. Atmospheric breakup of terrestrial impactors // Proc. Lunar Planet. Sci. 1981. V. 12A. Pp. 29-35.

4. Chyba C.F., Thomas P.J., Zahnle K.J. The 1908 Tunguska explosion - Atmospheric disruption of a stony asteroid // Nature. 1993. V. 361. Pp. 40-44.

5. Hills J.G., Goda M.P. The fragmentation of small asteroids in the atmosphere// Astron. J. 1993. V. 105. № 3. Pp. 1114-1144.

6. Григорян С.С., Ибодов Ф.С., Ибадов С.И. Челябинский суперболид: к физике взрыва // Астрон. вестн. 2013. Т. 47. № 4. С. 292-298.

7. Register P.J., Mathias D.L., Wheeler L.F. Asteroid fragmentation approaches for modeling atmospheric energy deposition // Icarus. 2017. V. 284. Pp. 157-166.

8. Collins G.S., Lynch E., McAdam R., Davison T.M. A numerical assessment of simple airblast models of impact airbursts // Meteorit. \& Planet. Sci. 2017. V. 52. Pp. 1542-1560.

9. Брыкина И.Г. О модели фрагментации крупного метеороида: моделирование взаимодействия Челябинского метеороида с атмосферой // Астрон. вестн. 2018. Т. 52. № 5. С. 437-446.

10. McMullan S., Collins G.S. Uncertainty Quantification in Continuous Fragmentation Airburst Models // Icarus. 2019. V. 327. Pp. 19-35.

11. Borovička J., Popova O. Spurný P. The Maribo CM2 meteorite fall—Survival of weak material at high entry speed// Meteorit. \& Planet. Sci. 2019. V. 54. Pp. 1024-1041.

12. Брыкина И.Г., Брагин М.Д., Егорова Л.А. О моделях фрагментации метеороидов в атмосфере // Физико-химическая кинетика в газовой динамике. 2019. Т. 20. Вып. 2.

http://chemphys.edu.ru/issues/2019-20-2/articles/822/.

13. Голомазов М.М., Литвинов И.А., Литвинов Л.А., Иванков А.А., Финченко В.С. Численное моделирование обтекания спускаемых аппаратов при входе в атмосферу планеты // Вестн. МГТУ им. Н.Э. Баумана. Сер. “Машиностр.” 2011. № 4. С. 42-53.

14. Брыкина И.Г., Егорова Л.А. Аппроксимационные формулы для радиационного теплового потока при больших скоростях // Изв. РАН. МЖГ. 2019. № 4. С. 123-134.

15. Suttles J.T., Sullivan E.M., Margolis S.B. Curve fits of predicted inviscid stagnation-point radiative heating rates, cooling factors, and shock standoff distances for hyperbolic earth entry. NASA TN D7622. 1974. $45 \mathrm{p}$.

16. Johnston C.O., Mazaheri A., Gnoffo P., Kleb B., Sutton K., Prabhu D., Brandis A.M., Bose D. Radiative heating uncertainty for hyperbolic Earth entry, part 1: flight simulation modeling and uncertainty // J. Spacecraft \& Rockets. 2013. V. 50. No 1. Pp. 19-38.

17. Brown P.G., Assink J.D., Astiz L., Blaauw R., Boslough M.B., Borovička J., Brachet N., Brown D., Campbell-Brown M., Ceranna L., Cooke W., de Groot-Hedlin C., Drob D.P., Edwards W., Evers L.G., Garces M., Gill J., Hedlin M., Kingery A., Laske G., Le Pichon A., Mialle P., Moser D.E., Saffer A., Silber E., Smets P., Spalding R.E., Spurny P., Tagliaferri E., Uren D., Weryk R.J., Whitaker R., Krzeminski Z. A 500-kiloton airburst over Chelyabinsk and an enhanced hazard from small impactors. // Nature. 2013. V. 503. Pp. 238-241. 
18. Borovička J., Spurný P., Brown P., Wiegert P., Kalenda P., Clark, D.,Shrbený L. The trajectory, structure and origin of the Chelyabinsk asteroidal impactor// Nature. 2013. V. 503. Pp. 235-237.

19. Popova O.P., Jenniskens P., Emel'yanenko V., Kartashova A., Biryukov E., Khaibrakhmanov S., Shuvalov V., Rybnov Y., Dudorov A., Grokhovsky V.I., Badyukov D.D., Yin Q.-Z., Gural P.S., Albers J., Granvik M., Evers L.G., Kuiper J., Kharlamov V., Solovyov A., Rusakov Y.S., Korotkiy S., Serdyuk I., Korochantsev A.V., Larionov M.Yu., Glazachev D., Mayer E.M., Gisler G., Gladkovsky S.V., Wimpenny J., Snanborn M. E., Yamakawa A., Verosub K., Rowland D.J., Roeske S., Botto N. W., Friedrich J.M., Zolensky M., Le L., Ross D., Ziegler K. Nakamura T., Ahn I., Lee J.I., Zhou Q., Li Z.H., Liu Y., Tan G.-Q., Hiroi T., Sears D., Weinstein I. A., Vokhmintsev A.S., Ishchenko A.V., Schmitt-Kopplin P., Hertkorn N., Nagao K., Haba M. K., Komatsu M., Mikouchi T. Chelyabinsk Airburst, Damage Assessment, Meteorite Recovery, and Characterization // Science. 2013. V. 342. Iss. 6162. Pp. 1069-1073.

\section{References}

1. Maksimov, F. A., "Sverhzvukovoe obtekanie sistemy tel," Komp'juternye issledovanija i modelirovanie (Computer Research and Modeling), Vol. 5, 2013, pp. 969-980. (in Russian) https://doi.org/10.20537/2076-7633-2013-5-6-969-980

2. Grigorjan, S. S., "Meteorites motion and destruction in planet atmospheres," Cosmic researches, Vol. 17, 1979, pp. 724-740. http://adsabs.harvard.edu/abs/1980CosRe..17..724G

3. Melosh, H. J., "Atmospheric breakup of terrestrial impactors," Proc. Lunar Planet. Sci., Vol. 12A 1981, pp. 29-35. http://adsabs.harvard.edu/full/1981mrbf.conf...29M

4. Chyba, C. F., Thomas, P. J., Zahnle, K. J., "The 1908 Tunguska explosion - Atmospheric disruption of a stony asteroid," Nature, Vol. 361, 1993, pp. 40-44. https://www.nature.com/articles/361040a0

5. Hills, J. G., Goda, M. P., "The fragmentation of small asteroids in the atmosphere," Astron. J., Vol. 105. No. 3, 1993, pp. 1114-1144. http://adsabs.harvard.edu/full/1993AJ...105.1114H

6. Grigorjan, S. S., Ibodov, F. S., Ibadov, S. I., "Physical mechanism of Chelyabinsk superbolideexplotion," Solar Syst. Res., Vol. 47, pp. 268-274. https://doi.org/10.1134/S0038094613040151

7. Register, P. J., Mathias, D. L., Wheeler, L. F., "Asteroid fragmentation approaches for modeling atmospheric energy deposition," Icarus, Vol. 284, 2017, pp. 157-166. http://doi.org/10.1016/j.icarus.2016.11.020.

8. Collins, G. S., Lynch, E., McAdam, R., Davison, T. M., "A numerical assessment of simple airblast models of impact airbursts," Meteorit. \& Planet. Sci., Vol. 52, 2017, pp. 1542-1560. https://doi.org/10.1111/maps.12873

9. Brykina, I. G., "Large meteoroid fragmentation: modeling the interaction of the Chelyabinsk meteoroid with the atmosphere," Solar Syst. Res., Vol. 52, 2018, pp. 426-434.

https://doi.org/10.1134/S0038094618050027

10. McMullan, S., Collins, G. S., "Uncertainty Quantification in Continuous Fragmentation Airburst Models," Icarus, Vol. 327, 2019, pp. 19-35. https://doi.org/10.1016/j.icarus.2019.02.013

11. Borovička, J., Popova, O. Spurný, P., "The Maribo CM2 meteorite fall-Survival of weak material at high entry speed," Meteorit. \& Planet. Sci., Vol. 54, 2019, pp. 1024-1041.

https://doi.org/10.1111/maps.13259

12. Brykina, I. G., Bragin, M. D., Egorova, L. A., "O modeljah fragmentacii meteoroidov v atmosphere," Fiziko-himicheskaja kinetika v gazovoj dinamike (Phisical-Chemical Kinetics in Gas Dynamics), Vol. 20, No. 2, 2019. (in Russian) http://chemphys.edu.ru/issues/2019-20-2/articles/822/

13. Golomazov, M. M., Litvinov, I. A., Litvinov, L. A., Ivankov, A. A., Finchenko, V. S., "Numerical simulation of flow past descent vehicles during planetary entry," Herald of the Bauman Moscow state technical university, Mechanical engineering, Vol. 4, No. 85, 2011, pp. 42-53. http://vestnikmach.ru/eng/catalog/simul/hidden/57.html 
14. Brykina, I. G., Egorova, L. A., "Approximation formulas for the radiative heat flux at high velocities," Fluid Dynamics), Vol. 54, 2019, pp. 562-574. https://doi.org/10.1134/S0015462819040037

15. Suttles, J. T., Sullivan, E. M., Margolis, S. B., "Curve fits of predicted inviscid stagnation-point radiative heating rates, cooling factors, and shock standoff distances for hyperbolic earth entry," NASA TN D-7622, 1974. https://ntrs.nasa.gov/archive/nasa/casi.ntrs.nasa.gov/19740021216.pdf

16. Johnston, C. O., Mazaheri, A., Gnoffo, P., Kleb, B., Sutton, K., Prabhu, D., Brandis, A. M., Bose, D., "Radiative heating uncertainty for hyperbolic Earth entry, part 1: flight simulation modeling and uncertainty," J. Spacecraft \& Rockets, Vol. 50, No. 1, 2013, pp. 19-38. https://doi.org/10.2514/1.A32254

17. Brown, P. G., Assink, J. D., Astiz, L., Blaauw, R., Boslough, M. B., Borovička, J., Brachet, N., Brown, D., Campbell-Brown, M., Ceranna, L., Cooke, W., de Groot-Hedlin, C., Drob, D.P., Edwards, W., Evers, L. G., Garces, M., Gill, J., Hedlin, M., Kingery, A., Laske, G., Le Pichon, A., Mialle, P., Moser, D. E., Saffer, A., Silber, E., Smets, P., Spalding, R. E., Spurny, P.,Tagliaferri, E., Uren, D., Weryk, R. J., Whitaker, R., Krzeminski, Z., "A 500-kiloton airburst over Chelyabinsk and an enhanced hazard from small impactors," Nature, Vol. 503, 2013, pp. 238-241.

https://doi.org/10.1038/nature12741

18. Borovička, J., Spurný, P., Brown, P., Wiegert, P., Kalenda, P., Clark, D., Shrbený, L., “The trajectory, structure and origin of the Chelyabinsk asteroidal impactor," Nature, Vol. 503, 2013, pp. 235-237. https://doi.org/10.1038/nature12671

19. Popova, O. P., Jenniskens, P., Emel'yanenko, V., Kartashova, A., Biryukov, E., Khaibrakhmanov, S., Shuvalov, V., Rybnov, Y., Dudorov, A., Grokhovsky, V. I., Badyukov, D. D., Yin, Q.-Z., Gural, P. S., Albers, J., Granvik, M., Evers, L. G., Kuiper, J., Kharlamov, V., Solovyov, A., Rusakov, Y. S., Korotkiy, S., Serdyuk, I., Korochantsev, A. V., Larionov, M. Yu., Glazachev, D., Mayer, E. M., Gisler, G., Gladkovsky, S. V., Wimpenny, J., Snanborn, M. E., Yamakawa, A., Verosub, K., Rowland, D. J., Roeske, S., Botto, N. W., Friedrich, J. M., Zolensky, M., Le, L., Ross, D., Ziegler, K. Nakamura, T., Ahn, I., Lee, J. I., Zhou, Q., Li, Z.-H., Liu, Y., Tan, G.-Q., Hiroi, T., Sears, D., Weinstein, I. A., Vokhmintsev, A. S., Ishchenko, A. V., Schmitt-Kopplin, P., Hertkorn, N., Nagao, K., Haba, M. K., Komatsu, M., Mikouchi, T., "Chelyabinsk Airburst, Damage Assessment, Meteorite Recovery, and Characterization," Science, Vol. 342, No.6162, 2013, pp. 1069-1073. https://doi.org/10.1126/science. 1242642 